\title{
Axiomatizations for the Shapley-Shubik power index for games with several levels of approval in the input and output
}

\author{
Sascha Kurz, Issofa Moyouwou ${ }^{\dagger}$ and Hilaire Touyem ${ }^{\ddagger}$
}

\begin{abstract}
The Shapley-Shubik index is a specialization of the Shapley value and is widely applied to evaluate the power distribution in committees drawing binary decisions. It was generalized to decisions with more than two levels of approval both in the input and the output. The corresponding games are called $(j, k)$ simple games. Here we present a new axiomatization for the Shapley-Shubik index for $(j, k)$ simple games as well as for a continuous variant, which may be considered as the limit case.

Key words: simple games, several levels of approval, Shapley-Shubik index, power indices, axiomatization, interval decisions

Math. Subj. Class. (2010): Primary 91A40, 91B12; Secondary 91A80, 91A12.

JEL Class.: C71, D70, D71.
\end{abstract}

\section{Introduction}

In [18] Shapley introduced a function that could be interpreted as the expected utility of a game from each of its positions via the axiomatic approach - the so-called Shapley value. A bit later, see [19], it was restricted to games with binary decisions, i.e., simple games. An axiomatization of this so-called Shapley-Shubik index was given quite a few years later by Dubey [3]. Nowadays, the Shapley-Shubik index is one of the most established power indices for committees drawing binary decisions. However, not all decisions are binary. Abstaining from a vote might be seen as a third option for the committee members. In general, there might also be any number $j \geq 2$ of alternatives that can be chosen from. To

\footnotetext{
* Department of Mathematics, Physics and Computer Science, University of Bayreuth, 95440 Bayreuth, Germany Tel.: +49-921-557353 Fax: +49-921-557352. E-mail: sascha.kurz@uni-bayreuth.de

${ }^{\dagger}$ Advanced Teachers Training College, University of Yaounde I, PO Box 47 Yaounde, Cameroon

${ }^{\ddagger}$ Research and Training Unit for Doctorate in Mathematics, Computer Sciences and Applications, University of Yaounde I, PO Box 812, Yaounde, Cameroon
} 
this end, simple games were generalized to $(j, k)$ simple games [7], where $j$ is the number of alternatives in the input, i.e., the voting possibilities, and $k$ the number of alternatives for the group decision. A Shapley-Shubik index for these $(j, k)$ simple games was introduced in [5] generalizing earlier attempts for special cases, see e.g. [4, pp. 291-293]. However, also other variants have been introduced in the literature, see e.g. $[2,8,10]$. Here, we will only consider the variant from [5]. A corresponding axiomatizations is given in [6].

If we normalize the input and output levels to numbers between zero and one, we can consider the limit if $j$ and $k$ tend to infinity for $(j, k)$ simple games. More precisely we can consider the input levels $i /(j-1)$ for $0 \leq i \leq j-1$ and the output levels $i /(k-1)$ for $0 \leq i \leq k-1$. Then those games are discrete approximations for games with input and output levels freely chosen from the real interval $[0,1]$. The later games were called simple aggregation functions in [13], linking to the literature on aggregation functions [9], and interval simple games in [14]. A Shapley-Shubik like index for those games was motivated and introduced in [12], an axiomatization is given in [14].

The success story of the Shapley-Shubik index for simple games, initiated by [18] and [19], triggered a huge amount of modifications and generalizations to different types of games, see e.g. [1] for some current research directions. We think that the variants from [5], for $(j, k)$-simple games, and from [12], for interval simple games, form one consistent way to generalize the Shapley-Shubik index for simple games. Here we mainly focus on an axiomatic justification, see our main result in Theorem 5.1. Moreover, we present another formula for the Shapley-Shubik index for $(j, k)$ simple games which is better suited for computation issues, see Lemma 3.1 and Theorem 4.1. For a generalization of the Banzhaf index a similar result was obtained in [17]. As the title of the preface of [1] names it, the idea of the Shapley value is the root of a still ongoing research agenda.

The remaining part of this paper is organized as follows. In Section 2 we introduce the necessary preliminaries and present the first few basic results. A Shapley-Shubik index $\Phi$ for general $(j, k)$ simple games is introduced in Section 3. Moreover, we study the first basic properties of $\Phi$. In Section 4 we introduce the average game, which is a TU game associated to each $(j, k)$ simple game. This notion is then used to formulated the new axiom of average convexity, which culminates in an axiomatic characterization of $\Phi$ in Section 5. In Section 6 we transfer all notions and the axiomatic characterization to interval simple games. 


\section{Preliminaries}

Let $N=\{1,2, \ldots, n\}$ be a finite set of voters. Any subset $S$ of $N$ is called a coalition and the set of all coalitions of $N$ is denoted by the power set $2^{N}$. For given integers $j, k \geq 2$ we denote by $J=\{0, \ldots, j-1\}$ the possible input levels and by $K=\{0, \ldots, k-1\}$ the possible output levels, respectively. We write $x \leq y$ for $x, y \in \mathbb{R}^{n}$ if $x_{i} \leq y_{i}$ for all $1 \leq i \leq n$. For each $\emptyset \subseteq S \subseteq N$ we write $x_{S}$ for the restriction of $x \in \mathbb{R}^{n}$ to $\left(x_{i}\right)_{i \in S}$. As an abbreviation, we write $x_{-S}=x_{N \backslash S}$. Instead of $x_{\{i\}}$ and $x_{-\{i\}}$ we write $x_{i}$ and $x_{-i}$, respectively. Slightly abusing notation we write $\mathbf{a} \in \mathbb{R}^{n}$, for the vector that entirely consists of $a$ 's, i.e., $\mathbf{0}$ for the zero vector.

A simple game with player set $N$ is a mapping $v: 2^{N} \rightarrow\{0,1\}$ with $v(\emptyset)=1, v(N)=1$, and $v(S) \leq v(T)$ for all $\emptyset \subseteq S \subseteq T \subseteq N$. Coalitions $S \subseteq N$ with $v(S)=1$ are called winning and losing otherwise. The interpretation in the voting context is as follows. Those elements $i \in N$, called voters or players, that are contained in a coalition $S$ are those that are in favor of a certain proposal. The other voters, i.e., those in $N \backslash S$, are against the proposal. If $v(S)=1$ then the proposal is implemented and otherwise the status quo persists. A simple game $v$ is weighted if there exists a quota $q \in \mathbb{R}_{>0}$ and weights $w_{i} \in \mathbb{R}_{\geq 0}$ for all $i \in N$ such that $v(S)=1$ iff $w(S):=\sum_{i \in S} w_{i} \geq q$. As notation we use $\left[q ; w_{1}, \ldots w_{n}\right]$ for a weighted (simple) game. An example is given by $v=[4 ; 3,2,1,1]$ with winning coalitions $\{1,2\}$, $\{1,3\},\{1,4\},\{1,2,3\},\{1,2,4\},\{1,3,4\},\{1,2,3,4\}$, and $\{2,3,4\}$. A simple game $v$ is a unanimity game if there exists a coalition $\emptyset \neq T \subseteq N$ such that $v(S)=1$ iff $T \subseteq S$. As an abbreviation we use the notation $\gamma_{T}$ for a unanimity game with defining coalition $T$. It is well known that each simple game admits a representation as disjunctions of a finite list of unanimity games. Calling a winning coalition minimal if all proper subsets are losing, such a list is given by the minimal winning coalitions, i.e., by $\{1,2\},\{1,3\},\{1,4\}$, and $\{2,3,4\}$ in the above example.

If being part of a coalition is modeled as voting "yes" and "no" otherwise, represented as 1 and 0 , respectively, then one can easily reformulate and generalize the definition of a simple game:

Definition 2.1. $A(j, k)$ simple game for $n$ players, where $j, k \geq 2$ and $n \geq 1$ are integers, is a mapping $v: J^{n} \rightarrow K$ with $v(\mathbf{0})=0, v(\mathbf{j}-\mathbf{1})=k-1$, and $v(x) \leq v(y)$ for all $x, y \in J^{n}$ with $x \leq y$. The set of all $(j, k)$ simple games on $N$ is denoted by $\mathcal{U}_{n}^{j, k}$ or by $\mathcal{U}_{n}$, whenever $j$ and $k$ are clear from the context.

So, $(2,2)$ simple games are in one-to-one correspondence to simple games. We use the usual ordering of $J$ (and $K$ ) as a set of integers, i.e., $0<1<\cdots<j-1$. In words, in the input set, 0 is the lowest level of approval, followed by 1 and so on. In general, we call a function $f: \mathbb{R}^{n} \supseteq U \rightarrow \mathbb{R}$ monotone if we have $f(x) \leq f(y)$ for all $x, y \in U$ with $x \leq y$. We remark that in [5] the author considers a more general definition of a $(j, k)$ simple game than we have presented here. Additionally the $j$ input levels 
and the $k$ output levels are given by a so-called numeric evaluation. Our case is called uniform numeric evaluation there, which motivated the notation $\mathcal{U}_{n}$ for $(j, k)$ simple games for $n$ players. We also call a vector $x \in J^{n}$ a profile.

Definition 2.2. Given a $(j, k)$ simple game $v$ with player set $N$, we call a player $i \in N$ a null player if $v(x)=v\left(x_{-i}, y_{i}\right)$ for all $x \in J^{n}$ and all $y_{i} \in J$. Two players $i, h \in N$ are called equivalent if $v(x)=v\left(x^{\prime}\right)$ for all $x, x^{\prime} \in J^{n}$ with $x_{l}=x_{l}^{\prime}$ for all $l \in N \backslash\{i, h\}, x_{i}=x_{h}^{\prime}$, and $x_{h}=x_{i}^{\prime}$.

In words, a player $i$ is a null player if its input $x_{i}$ does not alter the output $v(x)$. If interchanging the input $x_{i}$ and $x_{h}$ of two players does never alter the output $v(x)$, then players $i$ and $h$ are equivalent. By $\pi_{i h}$ we denote the transposition on $N$ interchanging $i$ and $h$, so that the previous condition reads $v(x)=v\left(\pi_{i h} x\right)$ for all $x \in J^{n}$. By $\mathcal{S}_{n}$ we denote the set of permutations of length $n$, i.e., the bijections on $N$.

Now let us introduce a subclass of $(j, k)$ simple games with the property that for each profile $x$, the collective decision $v(x)$ is either 0 (the lowest level of approval) or it is $k-1$ (the highest level of approval) depending on whether some given voters report some minimum approval levels. For example, when any full support of the proposal necessitates a full support of each voter in a given coalition $S$, players in $S$ are each empowered with a veto. One may require from each player in $S$ only a certain level of approval for a full support of the proposal. All such games will be called $(j, k)$ simple games with point-veto.

Definition 2.3. $A(j, k)$ simple game with a point-veto is a $(j, k)$ simple game $v$ such that there exists some $a \in J^{n} \backslash\{\mathbf{0}\}$ satisfying $v(x)=k-1$ if $a \leq x$ and $v(x)=0$ otherwise for all $x \in J^{n}$. In this case, $a$ is the veto and the game $v$ is denoted by $u^{a}$. For each coalition $S \in 2^{N}$ we abbreviate $w^{S}=u^{a}$, where $a_{i}=j-1$ for all $i \in S$ and $a_{i}=0$ otherwise.

We remark that $(2,2)$ simple games with a point veto are in one-to-one correspondence to the subclass of unanimity games within simple games. The set of all players who report a non-null approval level is denoted by $N^{a}$, i.e., $N^{a}=\left\{i \in N: 0<a_{i} \leq j-1\right\}$. Every player in $N^{a}$ will be called a vetoer of the game $u^{a}$. Note that for the vector $a$ defined via $w^{S}=u^{a}$ we have $N^{a}=S$.

Null players as well as equivalent players can be identified easily in a given $(j, k)$ simple game with point-veto:

Proposition 2.1. Let $a \in J^{n} \backslash\{0\}$. A player $i \in N$ is a null player of $u^{a}$ iff $i \in N \backslash N^{a}$. Two players $i, h \in N$ are equivalent in $u^{a}$ iff $a_{i}=a_{h}$. 
Proof. For every $a \in J^{n} \backslash\{\mathbf{0}\}$ and every $i \in N \backslash N^{a}$ we have $a_{i}=0$ by the definition of $N^{a}$. Now let $i \in N \backslash N^{a}$. For every $x \in J^{n}$ and every $y_{i} \in J$ we have $a \leq x$ iff $a \leq\left(x_{-i}, y_{i}\right)$. Thus, $u^{a}(x)=u^{a}\left(x_{-i}, y_{i}\right)$ and $i$ is a null player in $u^{a}$. Now let $i \in N^{a}$, i.e., $a_{i}>0$. Since $v(a)=k-1 \neq 0=v\left(a_{-i}, \mathbf{0}_{i}\right)$, player $i$ is not a null player in $u^{a}$.

Assume that $a_{i}=a_{h}$ and consider an arbitrary $x \in J^{n}$. Then we have $a \leq x$ if and only if $a \leq \pi_{i h} x$. The definition of $u^{a}$ directly gives $u^{a}(x)=u^{a}\left(\pi_{i h} x\right)$, so that the players $i$ and $h$ are equivalent in $u^{a}$. Now suppose that the players $i$ and $h$ are equivalent in $u^{a}$. Since $a \leq a$, we obtain $u^{a}(a)=u^{a}\left(\pi_{i h} a\right)=k-1$. This implies that $a \leq \pi_{i h} a$. Therefore $a_{i} \leq a_{h}$ and $a_{h} \leq a_{i}$, that is $a_{i}=a_{h}$.

Note that $(j, k)$ simple games can be combined using the disjunction $(\vee)$ or the conjunction $(\wedge)$ operations to obtain new games.

Definition 2.4. Let $v^{\prime}$ and $v^{\prime \prime}$ be two $(j, k)$-simple games with player set $N$. By $v^{\prime} \vee v^{\prime \prime}$ we denote the $(j, k)$ simple game $v$ defined by $v(x)=\max \left\{v^{\prime}(x), v^{\prime \prime}(x)\right\}$ for all $x \in J^{n}$. Similarly, by $v^{\prime} \wedge v^{\prime \prime}$ we denote the $(j, k)$ simple game $v$ defined by $v(x)=\min \left\{v^{\prime}(x), v^{\prime \prime}(x)\right\}$ for all $x \in J^{n}$.

We remark that the defining properties of a $(j, k)$ simple game can be easily checked. This can be specialized to the subclass of $(j, k)$ simple games with point veto, i.e., $(j, k)$ simple games with point-veto can be combined using the disjunction $(\vee)$ or the conjunction $(\wedge)$ operations to obtain new games. To see this, consider a non-empty subset $E$ of $J^{n} \backslash\{\mathbf{0}\}$ and define the $(j, k)$ simple game denoted by $u^{E}$ by $u^{E}(x)=k-1$ if $a \leq x$ for some $a \in E$ and $u^{E}(x)=0$ otherwise, where $x \in J^{n}$ is arbitrary. Note that the notational simplification $u^{\{a\}}=u^{a}$, where $a \in J^{n} \backslash\{\mathbf{0}\}$, goes in line with Definition 2.3.

Proposition 2.2. Let $E$ and $E^{\prime}$ be two non-empty subsets of $J^{n} \backslash\{\mathbf{0}\}$. Then, we have $u^{E} \vee u^{E^{\prime}}=u^{E \cup E^{\prime}}$ and $u^{E} \wedge u^{E^{\prime}}=u^{E^{\prime \prime}}$, where $E^{\prime \prime}=\left\{c \in J^{n}: c_{i}=\max \left(a_{i}, b_{i}\right)\right.$ for some $a \in E$ and $\left.b \in E^{\prime}\right\}$.

Proof. In order to prove $u^{E} \vee u^{E^{\prime}}=u^{E \cup E^{\prime}}$ we consider an arbitrary $x \in J^{n}$. If $u^{E \cup E^{\prime}}(x)=k-1$, then there exists $a \in E \cup E^{\prime}$ such that, $a \leq x$. Therefore $u^{E}(x)=k-1$ or $u^{E^{\prime}}(x)=k-1$ and $\left(u^{E} \vee u^{E^{\prime}}\right)(x)=k-1$. Now suppose that $u^{E \cup E^{\prime}}(x)=0$. Then, for all $a \in E \cup E^{\prime}$ we have $a \not \leq x$. Since $E \subseteq E \cup E^{\prime}$ and $E^{\prime} \subseteq E \cup E^{\prime}$ we have $b \not \leq x$ and $c \not \leq x$ for all $b \in E$ and all $c \in E^{\prime}$. This implies that $u^{E}(x)=u^{E^{\prime}}(x)=0$ and $\left(u^{E} \vee u^{E^{\prime}}\right)(x)=0$. Thus, $u^{E} \vee u^{E^{\prime}}=u^{E \cup E^{\prime}}$.

Similarly, in order to prove $u^{E} \wedge u^{E^{\prime}}=u^{E^{\prime \prime}}$ we consider an arbitrary $x \in J^{n}$. If $u^{E^{\prime \prime}}(x)=k-1$, then there exists $c \in E^{\prime \prime}$ such that $c \leq x$. But, by definition of $E^{\prime \prime}, c=\max (a, b)$ for some $a \in E$ and $b \in E^{\prime}$, that is $a \leq c \leq x$ and $b \leq c \leq x$. Hence, $u^{E}(x)=u^{E^{\prime}}(x)=k-1$ and $\left(u^{E} \wedge u^{E^{\prime}}\right)(x)=k-1$. Now assume that $u^{E^{\prime \prime}}(x)=0$ and $\left(u^{E} \wedge u^{E^{\prime}}\right)(x) \neq 0$. By definition of $u^{E}$ and $u^{E^{\prime}}$, we have $\left(u^{E} \wedge u^{E^{\prime}}\right)(x)=k-1$. 
Thus, there exists $a \in E$ and $b \in E^{\prime}$ such that $a \leq x$ and $b \leq x$. It follows that $c=\max (a, b) \leq x$, which is a contradiction to $u^{E^{\prime \prime}}(x)=0$. This proves $u^{E} \wedge u^{E^{\prime}}=u^{E^{\prime \prime}}$.

For $(j, k)=(5,3)$ and $n=3$ an example is given by $E=\{(1,2,3),(2,1,2)\}, E^{\prime}=\{(4,1,1),(1,1,3)\}$. With this, $E^{\prime \prime}=\{(4,2,3),(1,2,3),(2,1,3),(4,1,2)\}$. Note that we may remove $(4,2,3)$ from that list since $(4,2,3) \geq(1,2,3)$ (or $(4,2,3) \geq(4,1,2))$.

Especially, Proposition 2.2 yields that every $(j, k)$ simple game of the form $u^{E}$ is a disjunction of some $(j, k)$ simple games with point-veto. So, each $(j, k)$ simple game of the form $u^{E}$ will be called a $(j, k)$ simple game with veto. In the game $u^{E}, E$ can be viewed as some minimum requirements (or thresholds) on the approval levels of voters' inputs for the full support of the proposal. It is worth noticing that $u^{E}$ is $\{0, k-1\}$-valued; the final decision at all profiles is either a no-support or a fullsupport. The set of all veto $(j, k)$ simple games on $N$ is denoted $\mathcal{V}_{n}$. Note that Proposition 2.2 shows that $\mathcal{V}_{n}$ is a lattice.

The sum of two $(j, k)$ simple games cannot be a $(j, k)$ simple game itself. However, we will show that each $(j, k)$ simple game is a convex combination of $(j, k)$ simple games with veto.

Definition 2.5. A convex combination of the games $v_{1}, v_{2}, \ldots, v_{p} \in \mathcal{U}_{n}$ is given by $v=\sum_{t=1}^{p} \alpha_{t} v_{t}$ for some non-negative numbers $\alpha_{t}$, where $t=1,2, \ldots, p$, that sum to 1 .

Note that not all convex combinations of $(j, k)$ simple games are $(j, k)$ simple games.

Proposition 2.3. For each $(j, k)$ simple game $v$ there exist a collection of positive numbers $\alpha_{t}$, where $t=1,2, \ldots, p$, that sum to 1 and a collection $F_{t}(v)$, where $t=1,2, \ldots, p$, of non-empty subsets of $J^{n}$ such that $v=\sum_{t=1}^{p} \alpha_{t} u^{F_{t}(v)}$.

Proof. Let $v \in \mathcal{U}_{n}$ and $\mathcal{F}(v)=\left\{x \in J^{n}, v(x)>0\right\}$. Since $J^{n}$ is finite and $v$ is monotone, the elements of $\mathcal{F}(v)$ can be labeled in such a way that $\mathcal{F}(v)=\left\{x^{1}, x^{2}, \ldots, x^{p}\right\}$, where $x^{p}=\mathbf{1}, v\left(x^{t}\right) \leq v\left(x^{t+1}\right)$ for all $1 \leq t<p$, and $t \leq s$ whenever $x^{t} \leq x^{s}$. Now, set $x^{0}=\mathbf{0}$ and $F_{t}(v)=\left\{x^{s}, t \leq s \leq p\right\}, \alpha_{t}=\frac{v\left(x^{t}\right)-v\left(x^{t-1}\right)}{k-1}$ for all $1 \leq t \leq p$. By our assumption on $x^{t}$ we have $\alpha_{t} \geq 0$ for all $1 \leq t \leq p$. Moreover, it can be easily checked that $\sum_{t=1}^{p} \alpha_{t}=\frac{v\left(x^{p}\right)-v\left(x^{0}\right)}{k-1}=1$. set $u=\sum_{t=1}^{p} \alpha_{t} u^{F_{t}(v)}$.

In order to prove that $v=u$, we consider an arbitrary $x \in J^{n}$. First suppose that $x \notin \mathcal{F}(v)$. Since $v$ is monotone, there is no $a \in \mathcal{F}(v)$ such that $a \leq x$. By definition, it follows that $v^{F_{t}(v)}(x)=0$ for all $t=1,2, \ldots, p$. Therefore $v(x)=u(x)=0$. Now suppose that $x \in \mathcal{F}(v)$. Then $x=x^{s}$ for some $s=1,2, \ldots, p$. It follows that for all $t=1,2, \ldots, p$ we have $v^{F_{t}(v)}(x)=k-1$ if $1 \leq t \leq s$ and $v^{F_{t}(v)}(x)=0$ otherwise. Therefore

$$
u(x)=\sum_{t=1}^{s} \alpha_{t}=\sum_{t=1}^{s}\left(\frac{v\left(x^{t}\right)-v\left(x^{t-1}\right)}{k-1} \cdot(k-1)\right)=v\left(x^{s}\right)=v(x) .
$$


Clearly, the game $v$ is a convex combination of the games $u^{F_{t}(v)}$, where $t=1,2, \ldots, p$.

Proposition 2.3 underlines the importance of $(j, k)$ simple games with veto, i.e., every $(j, k)$ simple game can be obtained from $(j, k)$ simple games with veto as a convex combination.

Now let us consider a continuous version of $(j, k)$ simple games normalized to the real interval $I:=[0,1]$ for the input as well as the output levels. Following [13] and using the name from [14], we call a mapping $v:[0,1]^{n} \rightarrow[0,1]$ an interval simple game if $v(\mathbf{0})=0, v(\mathbf{1})=1$, and $v(x) \leq v(y)$ for all $x, y \in[0,1]^{n}$ with $x \leq y$. Replacing $J$ by $[0,1]$ in Definition 2.2 we can transfer the concept of a null player and that of equivalent players to interval simple games.

\section{The Shapley-Shubik index for simple and $(j, k)$ simple games}

Since in a typical simple game $v$ not all players are equivalent, the question of influence of a single player $i$ on the final group decision $v(S)$ arises. Even if $v$ can be represented as a weighted game, i.e., $v=[q ; w]$, the relative individual influence is not always reasonably reflected by the weights $w_{i}$. This fact is well-known and triggered the invention of power indices, i.e., mappings from a simple game on $n$ players to $\mathbb{R}^{n}$ reflecting the influence of a player on the final group decision. One of the most established power indices is the Shapley-Shubik index [19]. It can be defined via

$$
\operatorname{SSI}_{i}(v)=\sum_{i \in S \subseteq N} \frac{(s-1) !(n-s) !}{n !} \cdot[v(S)-v(S \backslash\{i\})]
$$

for all players $i \in N$, where $s=|S|$. If $v(S)-v(S \backslash\{i\})=1$, then we have $v(S)=1$ and $v(S \backslash\{i\})=0$ in a simple game and voter $i$ is called a swing voter.

In [19] the authors have motivated the Shapley-Shubik index by the following interpretation. Assume that the $n$ voters row up in a line and declare to be part in the coalition of "yes"-voters. Given an ordering of the players, the player that first guarantees that a proposal can be put through is then called pivotal. Considering all $n$ ! orderings $\pi \in \mathcal{S}_{n}$ of the players with equal probability then gives a probability for being pivotal for a given player $i \in N$ that equals its Shapley-Shubik index. So we can rewrite Equation (1) to

$$
\operatorname{SSI}_{i}(v)=\frac{1}{n !} \cdot \sum_{\pi \in \mathcal{S}_{n}}(v(\{j \in N: \pi(j) \leq \pi(i)\})-v(\{j \in N: \pi(j)<\pi(i)\})) .
$$

Setting $S_{\pi}^{i}:=\{j \in N: \pi(j) \leq \pi(i)\}$ we have $S_{\pi}^{i}=S$ for exactly $(s-1) !(n-s)$ ! permutations $\pi \in \mathcal{S}_{n}$ and an arbitrary set $\{i\} \subseteq S \subseteq N$, so that Equation (1) is just a simplification of Equation (2). 
Instead of assuming that all players vote "yes" one can also assume that all players vote "no". In [16] it is mentioned that the model also yields the same result if we assume that all players independently vote "yes" with a fixed probability $p \in[0,1]$. This was further generalized to probability measures $p$ on $\{0,1\}^{n}$ where vote vectors with the same number of "yes" votes have the same probability, see [11]. In other words, individual votes may be interdependent but must be exchangeable. That no further probability measures lead to the Shapley-Shubik index was finally shown in [15]. For the most symmetric case $p=\frac{1}{2}$ we can rewrite Equation (2) to

$$
\operatorname{SSI}_{i}(v)=\frac{1}{n ! \cdot 2^{n}} \cdot \sum_{(\pi, x) \in \mathcal{S}_{n} \times\{0,1\}^{n}} M(v,(\pi, x), i),
$$

where $M(v,(\pi, x), i)$ is one if player $i$ is pivotal for ordering $\pi$ and vote vector $x$ in $v$, see [15], and zero otherwise.

This line of reasoning can be used to motivate a definition of a Shapley-Shubik index for $(j, k)$ simple games as defined in [5], c.f. [12]. Suppose that voters successively and independently each choose a level of approval in $J$ with equal probability. Such a vote scenario is modeled by a roll-call $(\pi, x)$ that consists in a permutation $\pi$ of the voters and a profile $x \in J^{n}$ such for all $i \in N$, the integer $\pi(i) \in\{1,2, \ldots, n\}$ is the entry position of voter $i$ and $x_{i}$ is his approval level. Given an index $h \in\{1, \ldots, k-1\}$, a voter $i$ is an $h$-pivotal voter if the vote of player $i$, according to the ordering $\pi$ and the approval levels of his predecessors, pushes the outcome to at least $h$ or to at most $h-1$.

Example 3.1. Let $v$ be the $(3,3)$ simple game $v$ for 2 players defined by $v(0,0)=v(1,0)=0, v(1,1)=$ $v(0,1)=1$, and $v(2,0)=v(0,2)=v(2,1)=v(1,2)=v(2,2)=2$. As an example, consider the ordering $\pi=(2,1)$, i.e., player 2 is first, and the vote vector $x=(2,1)$. Before player 2 announce his vote $x_{2}=1$ all outcomes in $K=\{0,1,2\}$ are possible. After the announcement the outcome 0 is impossible, since $v(0,1)=1$, while the outcomes 2 and 3 are still possible. Thus, player 2 is the 1-pivotal voter. Finally, after the announcement of $x_{1}=2$, the outcome is determined to be $v(2,1)=2$, so that player 1 is the 2-pivotal voter.

Going in line with the above motivation and the definition from [5], the Shapley-Shubik index for $(j, k)$ simple games is defined for all $v \in \mathcal{U}_{n}$ and for all $i \in N$ by:

$$
\Phi_{i}(v)=\frac{1}{n ! \cdot j^{n} \cdot(k-1)} \sum_{h=1}^{k-1} \mid\left\{(\pi, x) \in \mathcal{S}_{n} \times J^{n}: i \text { is an } h \text {-pivot for } \pi \text { and } x \text { in } v\right\} \mid .
$$

Since several different definitions of a Shapley-Shubik index for $(j, k)$-simple games have been introduced in the literature, we prefer to use the more inconspicuously notation $\Phi_{i}(v)$ instead of the more 
suggestive notation $\operatorname{SSI}_{i}(v)$. For the $(j, k)$ simple game $v$ from Example 3.1 we have

$$
\Phi(v)=\left(\Phi_{1}(v), \Phi_{2}(v)\right)=\left(\frac{5}{12}, \frac{7}{12}\right) .
$$

Hereafter, some properties of $\Phi$ are explored. To achieve this, we introduce further definitions and axioms for power indices on $(j, k)$ simple games. First of all, we simplify Equation (4) to a more handy formula.

Lemma 3.1. For each $(j, k)$ simple game $v \in \mathcal{U}_{n}$ and each player $i \in N$ we have

$$
\Phi_{i}(v)=\sum_{i \in S \subseteq N} \frac{(s-1) !(n-s) !}{n !} \cdot[C(v, S)-C(v, S \backslash\{i\})],
$$

where $s=|S|$ and

$$
C(v, T)=\frac{1}{j^{n}(k-1)} \cdot \sum_{x \in J^{n}}\left(v\left((\mathbf{j}-\mathbf{1})_{T}, x_{-T}\right)-v\left(\mathbf{0}_{T}, x_{-T}\right)\right)
$$

for all $T \subseteq N$.

Proof. For a given permutation $\pi \in \mathcal{S}_{n}$ and $i \in N$, we set $\pi_{<i}=\{j \in N: \pi(j)<\pi(i)\}, \pi_{\leq i}=$ $\{j \in N: \pi(j) \leq \pi(i)\}, \pi_{>i}=\{j \in N: \pi(j)>\pi(i)\}$, and $\pi_{\geq i}=\{j \in N: \pi(j) \geq \pi(i)\}$. With this, we can rewrite $n ! \cdot j^{n} \cdot(k-1)$ times the right hand side of Equation (4) to

$$
\sum_{(\pi, x) \in \mathcal{S}_{n} \times J^{n}}\left(\left[v\left(x_{\pi_{<i}},(\mathbf{j}-\mathbf{1})_{\pi_{\geq i}}\right)-v\left(x_{\pi_{<i}}, \mathbf{0}_{\pi_{\geq i}}\right)\right]-\left[v\left(x_{\pi_{\leq i}},(\mathbf{j}-\mathbf{1})_{\pi_{>i}}\right)-v\left(x_{\pi_{\leq i}}, \mathbf{0}_{\pi_{>i}}\right)\right]\right) .
$$

The interpretation is as follows. Since $v$ is monotone, before the vote of player $i$ exactly the values in $\left\{v\left(x_{\pi_{<i}}, \mathbf{0}_{\pi_{\geq i}}\right), \ldots, v\left(x_{\pi_{<i}},(\mathbf{j}-\mathbf{1})_{\pi_{\geq i}}\right)\right\}$ are still possible as final group decision. After the vote of player $i$ this interval eventually shrinks to $\left\{v\left(x_{\pi_{\leq i}}, \mathbf{0}_{\pi_{>i}}\right), \ldots, v\left(x_{\pi_{\leq i}},(\mathbf{j}-\mathbf{1})_{\pi_{>i}}\right)\right\}$. The difference in (7) just computes the difference between the lengths of both intervals, i.e., the number of previously possible outputs that can be excluded for sure after the vote of player $i$.

As in the situation where we simplified the Shapley-Shubik index of a simple game given by Equation (2) to Equation (1), we observe that it is sufficient to know the sets $\pi_{\geq i}$ and $\pi_{>i}$ for every permutation $\pi \in \mathcal{S}_{n}$. So we can condense all permutations that lead to the same set and can simplify the expression in (7) and obtain Equation (5).

While we think that the roll-call motivation stated above for Equation (4) is a valid justification on its own, we also want to pursue the more rigor path to characterize power indices, i.e., we want to give an axiomatization. A set of properties that are satisfied by the Shapley-Shubik index for simple games and uniquely characterize the index is given, e.g., in $[18,19]$. In order to obtain a similar result for $(j, k)$ simple games, we consider a power index $F$ as a map form $v$ to $\mathbb{R}^{n}$ for all $(j, k)$ simple games $v \in \mathcal{U}_{n}$. 
Definition 3.1. A power index $F$ for $(j, k)$ simple games satisfies

- Positivity $(P)$ if $F(v) \neq \mathbf{0}$ and $F_{i}(v) \geq 0$ for all $i \in N$ and all $v \in \mathcal{U}_{n}$;

- Anonymity $(A)$ if $F_{\pi(i)}(\pi v)=F_{i}(v)$ for all permutations $\pi$ of $N, i \in N$, and $v \in \mathcal{U}_{n}$, where $\pi v(x)=v(\pi(x))$ and $\pi(x)=\left(x_{\pi(i)}\right)_{i \in N} ;$

- Symmetry $(S)$ if $F_{i}(v)=F_{j}(v)$ for all $v \in \mathcal{U}_{n}$ and all voters $i, j \in N$ that are equivalent in $v$;

- Efficiency (E) if $\sum_{i \in N} F_{i}(v)=1$ for all $v \in \mathcal{U}_{n}$;

- the Null player property $(N P)$ if $F_{i}(v)=0$ for every null voter $i$ of an arbitrary game $v \in \mathcal{U}_{n}$;

- the transfer property $(\mathrm{T})$ if for all $u, v \in \mathcal{U}_{n}$ and all $i \in N$ we have $F_{i}(u)+F_{i}(v)=F_{i}(u \vee v)+$ $F_{i}(u \wedge v)$, where $(u \vee v)(x)=\max \{u(x), v(x)\}$ and $(u \wedge v)(x)=\min \{u(x), v(x)\}$ for all $x \in J^{n}$, see Definition 2.4 and Proposition 2.2;

- Convexity $(C)$ if $F(w)=\alpha F(u)+\beta F(v)$ for all $u, v \in \mathcal{U}_{n}$ and all $\alpha, \beta \in \mathbb{R}_{\geq 0}$ with $\alpha+\beta=1$, where $w=\alpha u+\beta v \in \mathcal{U}_{n}$;

- Linearity (L) if $F(w)=\alpha F(u)+\beta F(v)$ for all $u, v \in \mathcal{U}_{n}$ and all $\alpha, \beta \in \mathbb{R}$, where $w=\alpha u+\beta v \in \mathcal{U}_{n}$.

Note that $\alpha \cdot u+\beta \cdot v$ does not need to be a $(j, k)$ simple game for $u, v \in \mathcal{U}_{n}$, where $\alpha \cdot u$ is defined via $(\alpha \cdot u)(x)=\alpha \cdot u(x)$ for all $x \in J^{n}$ and all $\alpha \in \mathbb{R}$. We remark that, obviously, (L) implies (C) and (L) implies (T). Also (S) is implied by (A). Some of the properties of Definition 3.1 have been proven to be valid for $\Phi$ in [5]. However, for the convenience of the reader we give an extended result and a full proof next:

Proposition 3.1. The power index $\Phi$, defined in Equation (4), satisfies the axioms $(P),(A),(S),(E)$, $(N P),(T),(C)$, and $(L)$.

Proof. We use the notation from the proof of Lemma 3.1 and let $v$ be an arbitrary $(j, k)$ simple game with $n$ players.

For each $x \in J^{n}, \pi \in \mathcal{S}_{n}$, and $i \in N$, we have $v\left(x_{\pi_{<i}},(\mathbf{j}-\mathbf{1})_{\pi_{\geq i}}\right) \geq v\left(x_{\pi_{\leq i}},(\mathbf{j}-\mathbf{1})_{\pi_{>i}}\right)$ and $v\left(x_{\pi_{\leq i}}, \mathbf{0}_{\pi_{>i}}\right) \geq v\left(x_{\pi_{<i}}, \mathbf{0}_{\pi_{\geq i}}\right)$, so that $\Phi_{i}(v) \geq 0$ due to Equation (7). Since we will show that $\Phi$ is efficient, we especially have $\Phi(v) \neq \mathbf{0}$, so that $\Phi$ is positive. 
For any permutation $\pi \in \mathcal{S}_{n}$ and any $0 \leq h \leq n$ let $\pi \mid h:=\{\pi(i): 1 \leq i \leq h\}$, i.e., the first $h$ players in ordering $\pi$. Then, for any profile $x \in J^{n}$, we have

$$
\begin{aligned}
& \sum_{i=1}^{n}\left(v\left(x_{\pi_{<i}},(\mathbf{j}-\mathbf{1})_{\pi_{\geq i}}\right)-v\left(x_{\pi_{\leq i}},(\mathbf{j}-\mathbf{1})_{\pi_{>i}}\right)+v\left(x_{\pi_{\leq i}}, \mathbf{0}_{\pi_{>i}}\right)-v\left(x_{\pi_{<i}}, \mathbf{0}_{\pi_{\geq i}}\right)\right) \\
= & \sum_{h=1}^{n}\left(v\left(x_{\pi \mid h-1},(\mathbf{j}-\mathbf{1})_{-\pi \mid h-1}\right)-v\left(x_{\pi \mid h},(\mathbf{j}-\mathbf{1})_{-\pi \mid h}\right)\right)+\sum_{h=1}^{n}\left(v\left(x_{\pi \mid h}, \mathbf{0}_{-\pi \mid h}\right)-v\left(x_{\pi \mid h-1}, \mathbf{0}_{-\pi \mid h-1}\right)\right) \\
= & v\left(x_{\pi \mid 0},(\mathbf{j}-\mathbf{1})_{-\pi \mid 0}\right)-v\left(x_{\pi \mid n},(\mathbf{j}-\mathbf{1})_{-\pi \mid n}\right)+v\left(x_{\pi \mid n}, \mathbf{0}_{-\pi \mid n}\right)-v\left(x_{\pi \mid 0}, \mathbf{0}_{-\pi \mid 0}\right) \\
= & v((\mathbf{j}-\mathbf{1}))-v(x)+v(x)-v(\mathbf{0})=k-1-0=k-1,
\end{aligned}
$$

so that Equation (7) gives $\sum_{i=1}^{n} \Phi_{i}(v)=1$, i.e., $\Phi$ is efficient.

The definition of $\Phi$ is obviously anonymous, so that it is also symmetric. If player $i \in N$ is a null player and $\pi \in \mathcal{S}_{n}$ arbitrary, then $v\left(x_{\pi_{<i}}, \mathbf{0}_{\pi_{\geq i}}\right)=v\left(x_{\pi_{\leq i}}, \mathbf{0}_{\pi_{>i}}\right)$ and $v\left(x_{\pi_{<i}},(\mathbf{j}-\mathbf{1})_{\pi_{\geq i}}\right)=$ $v\left(x_{\pi_{\leq i}},(\mathbf{j}-\mathbf{1})_{\pi_{>i}}\right)$, so that $\Phi_{i}(v)=0$, i.e., $\Phi$ satisfies the null player property. Since Equation $(7)$ is linear in the involved $(j, k)$ simple game, $\Phi$ satisfies $(\mathrm{L})$ as well as $(\mathrm{C})$, which is only a relaxation. Since $x+y=\max \{x, y\}+\min \{x, y\}$ for all $x, y \in \mathbb{R}, \Phi$ also satisfies the transfer axiom (T).

Actually the proof of Proposition 3.1 is valid for a larger class of power indices for $(j, k)$ simple games. To this end we associate each vector $a \in J^{n}$ with the function $v_{a}$ defined by

$$
v_{a}(S)=\frac{1}{k-1} \cdot\left[v\left((\mathbf{j}-\mathbf{1})_{S}, a_{-S}\right)-v\left(\mathbf{0}_{S}, a_{-S}\right)\right]
$$

for all $S \subseteq N$. With this, we define the mapping $\Phi^{a}$ on $\mathcal{U}_{n}$ by

$$
\Phi_{i}^{a}(v)=\sum_{i \in S \subseteq N} \frac{(s-1) !(n-s) !}{n !}\left[v_{a}(S)-v_{a}(S \backslash\{i\}]\right.
$$

for all $i \in N$. We remark that it can be easily checked that $v_{a}$ is a TU game, c.f. Section 4.

Similar as in the proof of Lemma 3.1, we conclude:

Proposition 3.2. For every $a \in J^{n}$ such that $a_{i}=a_{j}$ for all $i, j \in N$, the mapping $\Phi^{a}$ the axioms $(P)$, $(A),(S),(E),(N P),(T),(C)$, and $(L)$.

While the Shapley-Shubik index for simple games is the unique power index that is symmetric, efficient, satisfies both the null player property and the transfer property, see [3], this result does not transfer to general $(j, k)$ simple games.

Proposition 3.3. When $j \geq 3$, there exists some $a \in J^{n}$ such that $\Phi^{a} \neq \Phi$.

Proof. Consider the $(j, k)$ simple game $u^{b}$ with point-veto $b=(1, j-1,0, \cdots, 0) \in J^{n}$ and let $a=$ $(j-2, j-2, \cdots, j-2) \in J^{n}$. From Equation (8) we conclude $\Phi^{a}\left(u^{b}\right)=(0,1,0, \cdots, 0)$. Using Equation (5) we easily compute $\Phi\left(u^{b}\right)=\left(\frac{1}{j}, \frac{j-1}{j}, 0, \cdots, 0\right) \neq \Phi^{a}$. 
We remark that the condition $j \geq 3$ is necessary in Proposition 3.3, since for $(2,2)$ simple games the roll-call interpretation of Mann and Shapley, see [16], for the Shapley-Shubik index for simple games yields $\Phi^{0}=\Phi^{1}=\Phi$.

\section{The average game of a $(j, k)$ simple game}

Equation (5) in Lemma 3.1 has the important consequence that $\Phi(v)$ equals the Shapley value of the TU game $C(v, \cdot)$, where a TU game is a mapping $v: 2^{N} \rightarrow \mathbb{R}$ with $v(\emptyset)=0$. To this end we introduce an operator that associates each $(j, k)$ simple game $v$ with a TU game $\widetilde{v}$ as follows.

Definition 4.1. Let $v \in \mathcal{U}_{n}$ be an arbitrary $(j, k)$ simple game. The average game, denoted by $\widetilde{v}$, associated to $v$ is defined by

$$
\widetilde{v}(S)=\frac{1}{j^{n}(k-1)} \sum_{x \in J^{n}}\left[v\left((\mathbf{j}-\mathbf{1})_{S}, x_{-S}\right)-v\left(\mathbf{0}_{S}, x_{-S}\right)\right]
$$

for all $S \subseteq N$.

With that notation our above remark reads:

Theorem 4.1. For every $(j, k)$ simple game $v$ the vector $\Phi(v)$ equals the Shapley value of $\widetilde{v}$.

For the $(j, k)$ simple game $v$ from Example 3.1 the average simple game is given by

$$
\tilde{v}(\emptyset)=0, \tilde{v}(\{1\})=\frac{1}{2}, \tilde{v}(\{2\})=\frac{2}{3} \text {, and } \tilde{v}(N)=1 .
$$

Before giving some properties of the average game operator we note that two distinct $(j, k)$ simple games may have the same average game, as illustrated in the following example.

Example 4.1. Consider the $(j, k)$ simple games $u, v \in \mathcal{U}_{n}$ defined by

- $u(x)=k-1$ if $x=\mathbf{1}$ and $u(x)=0$ otherwise;

- $v(x)=k-1$ if $x \neq \mathbf{0}$ and $v(x)=0$ otherwise

for all $x \in J^{n}$. Obviously, $u \neq v$. A simple calculation, using Equation (9), gives $\tilde{u}(S)=\tilde{v}(S)=\frac{1}{j^{n-s}}$ for all $S \in 2^{N}$.

The average game operator has some nice properties among which are the following:

Proposition 4.1. Given a $(j, k)$ simple game $v \in \mathcal{U}_{n}$,

(a) $\widetilde{v}$ is a TU game on $N$ that is $[0,1]$-valued and monotone; 
(b) any null player in $v$ is a null player in $\widetilde{v}$;

(c) any two equivalent players in $v$ are equivalent in $\widetilde{v}$;

(d) if $v=\sum_{t=1}^{p} \alpha_{t} v_{t}$ is a convex combination for some $v_{1}, \ldots, v_{p} \in \mathcal{U}_{n}$, then $\widetilde{v}=\sum_{t=1}^{p} \alpha_{t} \widetilde{v_{t}}$.

Proof. Let $v \in \mathcal{U}_{n}$. All mentioned properties of $\widetilde{v}$ are more or less transfered from the corresponding properties of $v$ via Equation (9). More precisely:

(a) Note that $\widetilde{v}(\emptyset)=\frac{1}{j^{n}(k-1)} \sum_{x \in J^{n}}[v(x)-v(x)]=0$ and $\widetilde{v}(N)=\frac{1}{j^{n}(k-1)} \sum_{x \in J^{n}}[v((\mathbf{j}-\mathbf{1}))-v(\mathbf{0})]=$ 1. Since $v$ is monotone and $\mathbf{0} \leq x \leq(\mathbf{j}-\mathbf{1})$ for all $x \in J^{n}$, we have $v\left((\mathbf{j}-\mathbf{1})_{S}, x_{-S}\right) \leq$ $v\left((\mathbf{j}-\mathbf{1})_{S}, x_{-T}\right)$ and $v\left(\mathbf{0}_{S}, x_{-S}\right) \geq v\left(\mathbf{0}_{S}, x_{-T}\right)$ for all $\emptyset \subseteq S \subseteq T \subseteq N$. Thus, we can conclude $0 \leq \widetilde{v}(S) \leq \widetilde{v}(T) \leq 1$ from Equation $(9)$

(b) Let $i \in N$ be a null player in $v$ and $S \subseteq N \backslash\{i\}$. Since $v\left((\mathbf{j}-\mathbf{1})_{S \cup\{i\}}, x_{-(S \cup\{i\})}\right)=v\left((\mathbf{j}-\mathbf{1})_{S}, x_{-S}\right)$ and $v\left(\mathbf{0}_{S \cup\{i\}}, x_{-(S \cup\{i\})}\right)=v\left(\mathbf{0}_{S}, x_{-S}\right)$, we have that $\widetilde{v}(S \cup\{i\})=\widetilde{v}(S)$, i.e., player $i$ is a null player in $\widetilde{v}$.

(c) Let $i, h \in N$ be two equivalent players in $v, S \subseteq N \backslash\{i, h\}$, and $\pi_{i h} \in \mathcal{S}_{n}$ the transposition that interchanges $i$ and $h$. Since $v\left((\mathbf{j}-\mathbf{1})_{S \cup\{i\}}, x_{-(S \cup\{i\})}\right)=v\left((\mathbf{j}-\mathbf{1})_{S \cup\{h\}},\left(\pi_{i h} x\right)_{-S \cup\{h\}}\right)$ and $v\left(\mathbf{0}_{S \cup\{i\}}, x_{-(S \cup\{i\})}\right)=v\left(\mathbf{0}_{S \cup\{h\}},\left(\pi_{i h} x\right)_{-S \cup\{h\}}\right)$, we have $\widetilde{v}(S \cup\{i\})=\widetilde{v}(S \cup\{h\})$, i.e., players $i$ and $h$ are equivalent in $\widetilde{v}$.

(d) Now suppose that $v=\sum_{t=1}^{p} \alpha_{t} v_{t}$ is a convex combination for some $v_{1}, v_{2}, \cdots, v_{p} \in \mathcal{U}_{n}$. Since $v\left((\mathbf{j}-\mathbf{1})_{S}, x_{-S}\right)=\sum_{t=1}^{p} \alpha_{t} v_{t}\left((\mathbf{j}-\mathbf{1})_{S}, x_{-S}\right)$ and $v\left(\mathbf{0}_{S}, x_{-S}\right)=\sum_{t=1}^{p} \alpha_{t} v_{t}\left(\mathbf{0}_{S}, x_{-S}\right)$, Equation (9) gives $\widetilde{v}(S)=\sum_{t=1}^{p} \alpha_{t} \widetilde{v_{t}}(S)$ for all $\emptyset \subseteq S \subseteq N$.

The operator that associates each $(j, k)$ simple game $v$ with its average game $\widetilde{v}$ can be seen as a coalitional representation of $(j, k)$ simple games. Moreover, Proposition 4.1 suggests that this representation preserves some properties of the initial game. The average game of a $(j, k)$ simple game with a point-veto is provided by:

Proposition 4.2. Given $a \in J^{n} \backslash\{\mathbf{0}\}$, the average game $\widetilde{u^{a}}$ satisfies for every coalition $S \neq N$

$$
\widetilde{u^{a}}(S)=\left\{\begin{array}{cl}
\prod_{i \in N \backslash S}\left(\frac{j-a_{i}}{j}\right) & \text { if } S \cap N^{a} \neq \emptyset \\
0 & \text { if } S \cap N^{a}=\emptyset
\end{array}\right.
$$


Proof. Let $a \in J^{n} \backslash\{\mathbf{0}\}$ and $\emptyset \subsetneq S \subsetneq N$.

First suppose that $S \cap N^{a}=\emptyset$. Then, for all $x \in J^{n}$ we have $a \leq\left((\mathbf{j}-\mathbf{1})_{S}, x_{-S}\right)$ iff $a \leq\left(\mathbf{0}_{S}, x_{-S}\right)$. Thus, $u^{a}\left(\left((\mathbf{j}-\mathbf{1})_{S}, x_{-S}\right)\right)=u^{a}\left(\left(\mathbf{0}_{S}, x_{-S}\right)\right)$. It then follows from $(9)$ that $\widetilde{u^{a}}(S)=0$.

Now suppose that $S \cap N^{a} \neq \emptyset$. Then, for all $x \in J^{n}$ we have $a \not \leq\left(\mathbf{0}_{S}, x_{-S}\right)$. Thus, $u^{a}\left(\left(\mathbf{0}_{S}, x_{-S}\right)\right)=0$. Note that $a \leq\left((\mathbf{j}-\mathbf{1})_{S}, x_{-S}\right)$ iff $a_{-S} \leq x_{-S}$. Hence,

$$
\begin{aligned}
\widetilde{u^{a}}(S) & =\frac{1}{j^{n}(k-1)} \sum_{x \in J^{n}} u^{a}\left((\mathbf{j}-\mathbf{1})_{S}, x_{-S}\right)=\frac{1}{j^{n-s}(k-1)} \sum_{x_{-S} \in J^{-S}} u^{a}\left((\mathbf{j}-\mathbf{1})_{S}, x_{-S}\right) \\
& =\frac{1}{j^{n-s}(k-1)} \sum_{x_{-S} \in J^{-S} \wedge a_{-S} \leq x_{-S}} u^{a}\left((\mathbf{j}-\mathbf{1})_{S}, x_{-S}\right) \\
& =\frac{1}{k-1} \cdot(k-1) \frac{\left|\left\{x_{-S} \in J^{-S}, a_{-S} \leq x_{-S}\right\}\right|}{j^{n-s}}=\prod_{i \in N \backslash S}\left(\frac{j-a_{i}}{j}\right) .
\end{aligned}
$$

It may be interesting to check whether each $(j, k)$ simple game may be decomposed as a combination of $(j, k)$ simple game with a point-veto of the form $a \in\{0, j-1\}^{n}$. The response is affirmative when one considers combinations between average games. Before we prove this, recall that the average game associated with each $(j, k)$ simple game is a TU game on $N$. The set of all TU games on $N$ is vector space and a famous basis consists in all unanimity games $\left(\gamma_{S}\right)_{S \in 2^{N}}$, where $\gamma_{S}(T)=1$ if $S \subseteq T$ and $\gamma_{S}(T)=0$ otherwise. ${ }^{1}$

In Definition 2.3 we have introduced the notation $w^{S}=u^{a}$ for a coalition $S \in 2^{N}$, where $a \in J^{n}$ is specified by $a_{i}=j-1$ if $i \in S$ and $a_{i}=0$ otherwise.

Proposition 4.3. For every coalition $C \in 2^{N}$, there exists a collection of real numbers $\left(y_{S}\right)_{S \in 2^{C}}$ such that

$$
\widetilde{w^{C}}=\sum_{S \in 2^{C}} y_{S} \gamma_{S} .
$$

Proof. Note that $\widetilde{w^{C}}$ is a TU game on $N$. Therefore, for some real numbers $\left(y_{S}\right)_{S \in 2^{N}}$ we have

$$
\widetilde{w^{C}}=\sum_{S \in 2^{N}} y_{S} \gamma_{S}
$$

This proves the result for $C=N$. Now, suppose that $C \neq N$. Consider $\mathcal{E}_{k}=\left\{T \in 2^{N}, T \backslash C \neq\right.$ $\emptyset$ and $|T|=k\}$ for $1 \leq k \leq n$. We prove by induction on $k$ that $y_{T}=0$ for all coalitions $T \in \mathcal{E}_{k}$. More formally, consider the assertion $\mathcal{P}(k)$ : for all $T \in \mathcal{E}_{k}$, we have $y_{T}=0$.

\footnotetext{
${ }^{1}$ The definition of unanimity games has already been given in the second paragraph of Section 2.
} 
First assume that, $k=1$. Let $T \in \mathcal{E}_{k}$, then there exists $i \in N \backslash C$ such that $T=\{i\}$. Since player $i$ is not contained in $C$, Proposition 2.1 and Proposition 4.1 yield that $i$ is a null player in $\widetilde{w^{C}}$, so that $\widetilde{w^{C}}(T)=0$. Since Equation $(10)$ gives $\widetilde{w^{C}}(T)=\sum_{S \in 2^{T}} y_{S}=y_{T}$, we have $y_{T}=0$. Therefore $\mathcal{P}(1)$ holds. Now consider $2 \leq k \leq n$ suppose and $\mathcal{P}(l)$ holds for all $1 \leq l<k$. Let $T \in \mathcal{E}_{k}$, then there exists $i \in N \backslash C$ such that $T=K \cup\{i\}, i \notin K \neq \emptyset$. Since $i$ is a null player in $\widetilde{w^{C}}$, we have $\widetilde{w^{C}}(T)-\widetilde{w^{C}}(K)=0$. Using Equation (10) we compute:

$$
\widetilde{w^{C}}(T)-\widetilde{w^{C}}(T)=\sum_{S \in 2^{T}} y_{S}-\sum_{S \in 2^{K}} y_{S}=y_{T}+\sum_{i \in S \varsubsetneqq T} y_{S}=y_{T}
$$

using $S \backslash C \neq \emptyset$ and $1 \leq|S|<|T|=k$. Thus, we have $y_{T}=0$, which proves our claim.

Proposition 4.4. For every $(j, k)$ simple game $u \in \mathcal{U}_{n}$, there exists a collection of real numbers $\left(x_{S}\right)_{S \in 2^{N}}$ such that

$$
\widetilde{u}=\sum_{S \in 2^{N}} x_{S} \widetilde{w^{S}}
$$

Proof. The result is straightforward when $j=2$ since $J$ reduces to $J=\{0,1\}$. In the rest of the proof, we assume that $j \geq 3$. Note that all TU games on $N$ can be written as a linear combination of unanimity games $\left(\gamma_{S}\right)_{S \in 2^{N}}$. It is then sufficient to only prove that each TU game $\gamma_{C}$ for $C \in 2^{N}$ is a linear combination of the TU games $\left(\widetilde{w^{S}}\right)_{S \in 2^{C}}$. The proof is by induction on $1 \leq k=|C| \leq n$. More precisely, we prove the assertion $\mathcal{A}(k)$ that for all $C \in 2^{N}$ such that $|C| \leq k$, there exists a collection $\left(z_{S}\right)_{S \in 2^{C}}$ such that

$$
\gamma_{C}=\sum_{S \in 2^{C}} z_{S} \widetilde{w^{S}}
$$

First assume that $k=1$. Using Proposition 4.2, it can be easily checked that we have $\gamma_{\{i\}}=\widetilde{\left.w^{\{i}\right\}}$ for all $i \in N$. Therefore $\mathcal{A}(1)$ holds. Now, consider a coalition $C$ such that $|C|=k \in\{2, \ldots, n\}$ and assume that $\mathcal{A}(l)$ holds for all $l$ such that $1 \leq l<k$. By Proposition 4.3 , there exists some real numbers $\left(\alpha_{S}\right)_{S \in 2^{C}}$ and $\left(\beta_{S}\right)_{S \in 2^{C} \backslash\{C\}}$ such that

$$
\widetilde{w^{C}}=\sum_{S \in 2^{C}} \alpha_{S} \gamma_{S}=\alpha_{C} \gamma_{C}+\sum_{S \in 2^{C} \backslash\{C\}} \alpha_{S} \gamma_{S}=\alpha_{C} \gamma_{C}+\sum_{S \in 2^{C} \backslash\{C\}} \beta_{S} \widetilde{w^{S}}
$$

where the last equality holds by the induction hypothesis. Moreover, $\alpha_{C}$ can be determined using Proposition 4.2 for $c=|C|$ by:

$$
\alpha_{C}=\sum_{S \in 2^{C}}(-1)^{|C \backslash S|} \widetilde{w^{C}}(S)=\sum_{s=1}^{c}(-1)^{c-s}\left(\begin{array}{l}
c \\
s
\end{array}\right)\left(\frac{j-1}{j}\right)^{c-s}=\frac{1-(1-j)^{c}}{j^{c}} \neq 0 \text { since } j-1 \geq 2 .
$$

Therefore we get

$$
\gamma_{C}=\sum_{S \in 2^{C}} z_{S} \widetilde{w^{S}}
$$


where for all $S \in 2^{C}, z_{S}=-\frac{1}{\alpha_{C}}$ if $S=C$ and $z_{S}=-\frac{\beta_{S}}{\alpha_{C}}$ otherwise. This gives $\mathcal{A}(k)$. In summary, each $\gamma_{S}, S \in 2^{N}$ is a linear combination of $\widetilde{w^{C}}, C \in 2^{N}$. Thus, the proof is completed since $\widetilde{u}$ is a linear combination of $\gamma_{S}, S \in 2^{N}$.

Before we continue, note that by Equation (12), for $C \in 2^{N}$ each TU game $\gamma_{C}$ is a linear combination of the TU games $\left(\widetilde{w^{S}}\right)_{S \in 2^{N}}$. Since $\left(\gamma_{S}\right)_{S \in 2^{N}}$ is a basis of the vector space of all TU games on $N$, it follows that $\left(\widetilde{w^{S}}\right)_{S \in 2^{N}}$ is also a basis of the vector space of all TU games on $N$.

\section{A characterization of the Shapley-Shubik index for $(j, k)$ simple games}

As shown in Proposition 3.2 the axioms of Definition 3.1 are not sufficient to uniquely characterize the power index $\Phi$ for the class of $(j, k)$ simple games. Therefore we introduce an additional axiom.

Definition 5.1. A power index $F$ for $(j, k)$ simple games is averagely convex $(A C)$ if we always have

$$
\sum_{t=1}^{p} \alpha_{t} F\left(u_{t}\right)=\sum_{t=1}^{q} \beta_{t} F\left(v_{t}\right)
$$

whenever

$$
\sum_{t=1}^{p} \alpha_{t} \widetilde{u}_{t}=\sum_{t=1}^{q} \beta_{t} \widetilde{v_{t}}
$$

where $u_{1}, u_{2}, \ldots, u_{p}, v_{1}, v_{2}, \ldots, v_{q} \in \mathcal{U}_{n}$ and $\left(\alpha_{t}\right)_{1 \leq t \leq p},\left(\beta_{t}\right)_{1 \leq t \leq q}$ are non-negative numbers that sum to 1 each.

One may motivate the axiom (AC) as follows. In a game, the a priori strength of a coalition, given the profile of the other individuals, is the difference between the outputs observed when all of her members respectively give each her maximum support and her minimum support. The average strength game associates each coalition with her expected strength when the profile of other individuals uniformly varies. Average convexity for power indices is the requirement that whenever the average game of a game is a convex combination of the average games of two other games, then the same convex combination still applies for the power distributions.

We remark that the axiom of Average Convexity is much stronger than the axiom of Convexity. A minor technical point is that $\sum_{t=1}^{p} \alpha_{t} u_{t}$ as well as $\sum_{t=1}^{q} \beta_{t} v_{t}$ do not need to be $(j, k)$ simple games. However, the more important issue is that

$$
\widetilde{p} \sum_{t=1} \alpha_{t} u_{t} \stackrel{\text { Proposition 4.1.(d) }}{=} \sum_{t=1}^{p} \alpha_{t} \widetilde{u_{t}}=\sum_{t=1}^{q} \beta_{t} \widetilde{v_{t}} \stackrel{\text { Proposition }}{=} \stackrel{\widetilde{q} \text { 4.(d) }}{\sum_{t=1}^{q} \beta_{t} v_{t}} \text {, }
$$


i.e., Equation (14), is far less restrictive than

$$
\sum_{t=1}^{p} \alpha_{t} u_{t}=\sum_{t=1}^{q} \beta_{t} v_{t}
$$

since two different $(j, k)$ simple games may have the same average game, see Example 4.1. Further evidence is given by the fact that the parametric power indices $\Phi^{a}$, defined in Equation (8), do not all satisfy (AC).

Proposition 5.1. When $j \geq 3$, there exists some $a \in J^{n}$ such that $\Phi^{a}$ does not satisfy (AC).

Proof. As in the proof of Proposition 3.3, consider the $(j, k)$ simple game with point-veto $b=(1, j-$ $1,0, \cdots, 0) \in J^{n}$ and let $a=(j-2, j-2, \cdots, j-2)$. It can be easily checked that, for all subsets $T \subseteq N$ we have

$$
\widetilde{u^{b}}(T)=\left\{\begin{array}{cll}
1 & \text { if } & 1,2 \in T \\
(j-1) / j & \text { if } & 2 \in T \subseteq N \backslash\{1\} \\
1 / j & \text { if } & 1 \in T \subseteq N \backslash\{2\} \\
0 & \text { if } & T \subseteq N \backslash\{1,2\}
\end{array}\right.
$$

and that

$$
\widetilde{u^{b}}=\frac{1}{j} \cdot \widetilde{w^{\{1\}}}+\frac{j-1}{j} \cdot \widetilde{w^{\{2\}}}
$$

holds. Since $\Phi^{a}$ satisfies (NP), (E), (S) we can easily compute $\Phi^{a}\left(w^{\{1\}}\right)=(1,0, \cdots, 0)$ and $\Phi^{a}\left(w^{\{2\}}\right)=$ $(0,1,0, \cdots, 0)$. Therefore,

$$
\frac{1}{j} \cdot \Phi^{a}\left(w^{\{1\}}\right)+\frac{j-1}{j} \cdot \Phi^{a}\left(w^{\{2\}}\right)=\left(\frac{1}{j}, \frac{j-1}{j}, 0, \cdots, 0\right) .
$$

Using (8), one gets $\Phi^{a}\left(u^{b}\right)=(0,1,0, \cdots, 0)$. It then follows from equations (15) and (16) that $\Phi^{a}$ does not satisfy $(\mathrm{AC})$.

As a preliminary step to our characterization result in Theorem 5.1 we state:

Lemma 5.1. If a power index $F$ for the class $\mathcal{U}_{n}$ of $(j, k)$ simple games satisfies $(E)$, $(S)$, and $(N P)$, then we have $F\left(w^{C}\right)=\Phi\left(w^{C}\right)$ for all $C \in 2^{N}$.

Proof. Let $F$ be a power index on $\mathcal{U}_{n}$ that satisfies (E), (S), (NP) and let $C \in 2^{N}$ be arbitrary.

According to Proposition 2.1, all players $i, j \in C$ are equivalent in $w^{C}$ and those outside of $C$ are null players in the game $w^{C}$. Since both $F$ and $\Phi$ satisfy (E), (S), and (NP), we have $F_{i}\left(w^{C}\right)=\Phi_{i}\left(w^{C}\right)=\frac{1}{|C|}$ if $i \in C$ and $F_{i}\left(w^{C}\right)=\Phi_{i}\left(w^{C}\right)=0$ otherwise.

Theorem 5.1. A power index $F$ for the class $\mathcal{U}_{n}$ of $(j, k)$ simple games satisfies $(E)$, (S), (NP), and $(A C)$ if and only if $F=\Phi$. 
Proof. Necessity: As shown in Proposition 3.1, $\Phi$ satisfies (E), (S), and (NP). For (AC) the proof follows from Theorem 4.1 since the average game operator is linear by Proposition 4.1.

Sufficiency: Consider a power index $F$ for $(j, k)$ simple games that satisfies (E), (S), (NP), and (AC). Next, consider an arbitrary $(j, k)$ simple game $u \in \mathcal{U}_{n}$. By Proposition 4.4, there exists a collection of real numbers $\left(x_{S}\right)_{S \in 2^{N}}$ such that

$$
\widetilde{u}=\sum_{S \in 2^{N}} x_{S} \widetilde{w^{S}}=\sum_{S \in E_{1}} x_{S} \widetilde{w^{S}}+\sum_{S \in E_{2}} x_{S} \widetilde{w^{S}}
$$

where $E_{1}=\left\{S \in 2^{N}: x_{S}>0\right\}$ and $E_{2}=\left\{S \in 2^{N}: x_{S}<0\right\}$. Note that $E_{1} \neq \emptyset$ since $\widetilde{u}(N)=1$. As an abbreviation we set

$$
\varpi=\sum_{S \in E_{1}} x_{S} \widetilde{w^{S}}(N)=\sum_{S \in E_{1}} x_{S}>0
$$

It follows that

$$
\frac{1}{\varpi} \widetilde{u}+\sum_{S \in E_{2}} \frac{-x_{S}}{\varpi} \widetilde{w^{S}}=\sum_{S \in E_{1}} \frac{x_{S}}{\varpi} \widetilde{w^{S}} .
$$

Since (19) is an equality among two convex combinations, axiom (AC) yields

$$
\frac{1}{\varpi} F(u)+\sum_{S \in E_{2}} \frac{-x_{S}}{\varpi} F\left(w^{S}\right)=\sum_{S \in E_{1}} \frac{x_{S}}{\varpi} F\left(w^{S}\right) .
$$

Therefore by Lemma 5.1,

$$
\frac{1}{\varpi} F(u)+\sum_{S \in E_{2}} \frac{-x_{S}}{\varpi} \Phi\left(w^{S}\right)=\sum_{S \in E_{1}} \frac{x_{S}}{\varpi} \Phi\left(w^{S}\right) .
$$

Since $\Phi$ also satisfies (AC), we obtain

$$
\frac{1}{\varpi} F(u)+\sum_{S \in E_{2}} \frac{-x_{S}}{\varpi} \Phi\left(w^{S}\right)=\frac{1}{\varpi} \Phi(u)+\sum_{S \in E_{2}} \frac{-x_{S}}{\varpi} \Phi\left(w^{S}\right),
$$

so that $F(u)=\Phi(u)$.

Proposition 5.2. For $j \geq 3$, the four axioms in Theorem 5.1 are independent.

Proof. For each of the four axioms in Theorem 5.1, we provide a power index on $\mathcal{U}_{n}$ that meets the three other axioms but not the chosen one.

- The power index $2 \cdot \Phi$ satisfies (NP), (S), and (AC) but not (E).

- Denote by ED the equal division power index which assigns $\frac{1}{n}$ to each player for every $(j, k)$ simple game $v$. Then, the power index $\frac{1}{2} \cdot \Phi+\frac{1}{2} \cdot$ ED satisfies $(\mathrm{E}),(\mathrm{S})$ and $(\mathrm{AC})$, but not (NP). 
- In Proposition 3.2 we have constructed a parametric series of power indices that satisfiy (E), (S), and (NP). For $j \geq 3$, at least one example does not satisfy (AC), see Proposition 5.1.

- Recall that $\left(\widetilde{w^{S}}\right)_{S \in 2^{N}}$ is a basis of the vector space of all TU games on $N$. Thus given a $(j, k)$ simple game $u$, there exists a unique collection of real numbers $\left(x_{S}^{u}\right)_{S \in 2^{N}}$ such that

$$
\widetilde{u}=\sum_{S \in 2^{N}} x_{S}^{u} \widetilde{w^{S}}
$$

Consider some $i_{0} \in N$ and set

$$
F(u)=\sum_{S \in 2^{N}} x_{S}^{u} \cdot F\left(w^{S}\right)
$$

For each $S \in 2^{N} \backslash\{N\}$ we set $F_{i}\left(w^{S}\right)=\Phi\left(w^{S}\right)$. For $S=N$ we set $F_{i}\left(w^{N}\right)=\frac{2}{n+1}$ if $i=i_{0}$ and $F_{i}\left(w^{N}\right)=\frac{1}{n+1}$ otherwise. We can easily check that $F$ satisfies $(\mathrm{E}),(\mathrm{NP}),(\mathrm{AC})$, but not $(\mathrm{S})$.

This proves that the four axioms in Theorem 5.1 are independent.

\section{Axiomatization of the Shapley-Shubik index for interval simple games}

Similar as for $(j, k)$ simple games a Shapley-Shubik like index for interval simple games can be constructed from the idea of the roll-call model.

Definition 6.1. (cf. [12, Definition 6.2])

Let $v$ be an interval simple game with player set $N$ and $i \in N$ an arbitrary player. We set

$$
\begin{aligned}
\Psi_{i}(v)= & \frac{1}{n !} \sum_{\pi \in \mathcal{S}_{n}} \int_{0}^{1} \cdots \int_{0}^{1}\left[v\left(x_{\pi_{<i}}, \mathbf{1}_{\pi_{\geq i}}\right)-v\left(x_{\pi_{<i}}, \mathbf{0}_{\pi_{\geq i}}\right)\right] \\
& -\left[v\left(x_{\pi_{\leq i}}, \mathbf{1}_{\pi_{>i}}\right)-v\left(x_{\pi_{\leq i}}, \mathbf{0}_{\pi_{>i}}\right)\right] \mathrm{d} x_{1} \ldots \mathrm{d} x_{n} .
\end{aligned}
$$

In this section, we give a similar axiomatization for $\Psi$ (for interval simple games) as we did for $(j, k$ ) simple games and $\Phi$. By a power index for interval simple games we understand a mapping from the set of interval simple games for $n$ players to $\mathbb{R}^{n}$. Replacing $J$ by $I=[0,1]$ in Definition 3.1, allows us to directly transfer the properties of power indices for $(j, k)$ simple games to the present situation. Also Proposition 3.1 is valid for interval simple games and $\Psi$. More precisely, $\Psi$ satisfies (P), (A), (S), (E), (NP), and (T), see [13, Lemma 6.1]. The proof for (C) and (L) goes along the same lines as the proof of Proposition 3.1. Also the generalization of the power index to a parametric class can be done just as the one for $(j, k)$ simple games in Equation (8). 
Proposition 6.1. For every $\alpha \in[0,1]$ the mapping $\Psi^{a}$, where $a=(\alpha, \ldots, \alpha) \in[0,1]^{n}$, defined by

$$
\Psi_{i}^{a}(v)=\frac{1}{n !} \sum_{\pi \in \mathcal{S}_{n}}\left(\left[v\left(a_{\pi_{<i}}, \mathbf{1}_{\pi_{\geq i}}\right)-v\left(a_{\pi_{<i}}, \mathbf{0}_{\pi_{\geq i}}\right)\right]-\left[v\left(a_{\pi_{\leq i}}, \mathbf{1}_{\pi_{>i}}\right)-v\left(a_{\pi_{\leq i}}, \mathbf{0}_{\pi_{>i}}\right)\right]\right)
$$

for all $i \in N$, satisfies $(P),(A),(S),(E),(N P),(T),(C)$, and $(L)$.

Again, there exist vectors $a \in[0,1]^{n}$ and interval simple games $v$ with $\Psi^{a}(v) \neq \Psi(v)$. Also the simplified formula for $\Phi$ for $(j, k)$ simple games in Lemma 3.1 can be mimicked for interval simple games and $\Psi$, see [14].

Proposition 6.2. For every interval simple game $v$ with player set $N$ and every player $i \in N$ we have

$$
\Psi_{i}(v)=\sum_{i \in S \subseteq N} \frac{(s-1) !(n-s) !}{n !} \cdot[C(v, S)-C(v, S \backslash\{i\})],
$$

where $C(v, T)=\int_{[0,1]^{n}} v\left(\mathbf{1}_{T}, x_{-T}\right)-v\left(\mathbf{0}_{T}, x_{-T}\right) \mathrm{d} x$ for all $T \subseteq N$.

This triggers:

Definition 6.2. Let $v$ be an interval simple game on $N$. The average game associated with $v$ and denoted by $\widehat{v}$ is defined via

$$
\forall S \subseteq N, \widehat{v}(S)=\int_{I^{n}}\left[v\left(\mathbf{1}_{S}, x_{-S}\right)-v\left(\mathbf{0}_{S}, x_{-S}\right)\right] d x
$$

Theorem 6.1. For all every interval simple game $v$ on $N$ and for all $i \in N$,

$$
\Psi_{i}(v)=\sum_{i \in S \subseteq N} \frac{(s-1) !(n-s) !}{n !}[\widehat{v}(S)-\widehat{v}(S \backslash\{i\}]
$$

In other words, for a given interval simple game $v$ the power distribution $\Psi(v)$ is given by the Shapley value of its average game $\hat{v}$.

As with $(j, k)$ simple games, two distinct interval simple games may have the same average game as illustrated in the following example.

Example 6.1. Consider the interval simple games $u$ and $v$ defined on $N$ respectively for all $x \in[0,1]^{n}$ by : $u(x)=1$ if $x=\mathbf{1}$, and $u(x)=0$ otherwise; $v(x)=1$ if $x \neq \mathbf{0}$, and $v(x)=0$ otherwise. It is clear that, $u \neq v$. But, Equation (26) and a simple calculation give $\widehat{u}(S)=\widehat{v}(S)=1$ if $S=N$ and $\widehat{u}(S)=\widehat{v}(S)=0$ otherwise.

We can also transfer Proposition 4.1, i.e., the average game operator preserves the following nice properties of interval simple games. 
Proposition 6.3. For all $v \in \mathcal{C S G}_{n}, \widehat{v}$ is a $[0,1]$-valued $T U$ game on $N$ such that

(a) $\widehat{v}$ is a TU game on $N$ that is $[0,1]$-valued and monotone;

(b) any null voter in $v$ is null player in $\widehat{v}$;

(c) any two symmetric voters in $v$ are symmetric players in $\widehat{v}$;

(d) if $v=\sum_{t=1}^{p} \alpha_{t} v_{t}$ is a convex combination for some $v_{1}, \ldots, v_{p} \in \mathcal{U}_{n}$ then $\widehat{v}=\sum_{t=1}^{p} \alpha_{t} \widehat{v_{t}}$.

Proof. Very similar to the one of Proposition 4.1.

From Theorem 6.1 we can directly conclude that $\Psi$ also satisfies Average Convexity (AC), which is defined as in Definition 5.1.

For the remaining part of this section we introduce some further notation. For all $x \in I^{n}$, let $\mathbf{1}_{x}=\left\{i \in N, x_{i}=1\right\}$; and given a coalition $S$, let $C^{S}$ be the interval simple game defined for all $x \in I^{n}$ by $C^{S}(x)=1$ if $S \subseteq \mathbf{1}_{x}$ and $C^{S}(x)=0$ otherwise.

Proposition 6.4. For all $T \in 2^{N}, \widehat{C^{S}}=\gamma_{S}$.

Proof. Consider $S, T \subseteq N$. If $S \subseteq T$ then for all $x \in[0,1]^{n}, S \subseteq T \subseteq\left\{i \in N,\left(\mathbf{1}_{T}, x_{-T}\right)_{i}=1\right\}$ and $S \cap\left\{i \in N,\left(\mathbf{0}_{T}, x_{-T}\right)_{i}=1\right\}=\emptyset$. Then by definition of $C^{S}, C^{S}\left(\mathbf{1}_{T}, x_{-T}\right)=1$ and $C^{S}\left(\mathbf{0}_{T}, x_{-T}\right)=0$. Therefore,

$$
\widehat{C^{S}}(T)=\int_{[0,1[n}\left[C^{S}\left(\mathbf{1}_{T},, x_{-T}\right)-C^{S}\left(\mathbf{0}_{T}, x_{-T}\right)\right] d x=1=\gamma_{S}(T) .
$$

Now assume that $S \nsubseteq T$. Let $x \in[01)^{n}$. Note that $\left\{i \in N,\left(\mathbf{1}_{T}, x_{-T}\right)_{i}=1\right\}=T$ and $\{i \in$ $\left.N,\left(\mathbf{0}_{T}, x_{-T}\right)_{i}=1\right\}=\emptyset$. Therefore, $S \nsubseteq\left\{i \in N,\left(\mathbf{1}_{T}, x_{-T}\right)_{i}=1\right\}$ and $S \nsubseteq\left\{i \in N,\left(\mathbf{0}_{T}, x_{-T}\right)_{i}=1\right\}$. By the definition of $C^{S}$, it follows that $C^{S}\left(\mathbf{1}_{T}, x_{-T}\right)=C^{S}\left(\mathbf{0}_{T}, x_{-T}\right)=0$. Hence

$$
\widehat{C^{S}}(T)=\int_{\left[0,1\left[{ }^{n}\right.\right.}\left[C^{S}\left(\mathbf{1}_{T}, x_{-T}\right)-C^{S}\left(\mathbf{0}_{T}, x_{-T}\right)\right] d x=0=\gamma_{S}(T) .
$$

In both cases $\widehat{C^{S}}(T)=\gamma_{S}(T)$ for all $T \in 2^{N}$; that is $\widehat{C^{S}}=\gamma_{S}$.

Theorem 6.2. A power index $\Psi^{\prime}$ for interval simple games satisfies (E), (S), (NP) and (AC) if and only if $\Psi^{\prime}=\Psi$.

Proof.

Necessity: We have already remarked that $\Psi$ satisfies (E), (S), (AC), and (NP). 
Sufficiency: Let $\Psi^{\prime}$ be a power index for interval simple games on $N$ that simultaneously satisfies (E), (S), (AC), and (NP). Consider an interval simple game $u$. Note that $\widehat{u}$ is a TU game by Proposition 6.3. Thus by Proposition 6.4, there exists a collection of real numbers $\left(\alpha_{S}\right)_{S \in 2^{N}}$ such that

$$
\widehat{u}=\sum_{S \in 2^{N}} \alpha_{S} \cdot \widehat{C^{S}}=\sum_{S \in E_{1}} \alpha_{S} \cdot \widehat{C^{S}}+\sum_{S \in E_{2}} \alpha_{S} \cdot \widehat{C^{S}}
$$

where $E_{1}=\left\{S \in 2^{N}: \alpha_{S}>0\right\}$ and $E_{2}=\left\{S \in 2^{N}: \alpha_{S}<0\right\}$. Moreover, $E_{1} \neq \emptyset$ since $\widehat{v}(N)=1$. We set

$$
\varpi=\sum_{S \in E_{1}} \alpha_{S} \cdot \widehat{C^{S}}(N)=\sum_{S \in E_{1}} \alpha_{S}>0
$$

It follows that

$$
\frac{1}{\varpi} \widehat{u}+\sum_{S \in E_{2}} \frac{-\alpha_{S}}{\varpi} \widehat{C^{S}}=\sum_{S \in E_{1}} \frac{\alpha_{S}}{\varpi} \widehat{C^{S}} .
$$

Since (30) is an equality among two convex combinations, then by (AC), we deduce that

$$
\frac{1}{\varpi} \Psi^{\prime}(u)+\sum_{S \in E_{2}} \frac{-\alpha_{S}}{\varpi} \Psi^{\prime}\left(C^{S}\right)=\sum_{S \in E_{1}} \frac{\alpha_{S}}{\varpi} \Psi^{\prime}\left(C^{S}\right) .
$$

Note that given $S \in 2^{N}$, all voters in $S$ are equivalent in $C^{S}$ while all voters outside $S$ are null players in $C^{S}$. Since $\Psi^{\prime}$ and $\Psi$ satisfy $(\mathrm{E}),(\mathrm{S})$, and (NP), it follows that $\Psi^{\prime}\left(C^{S}\right)=\Psi\left(C^{S}\right)$. Thus,

$$
\frac{1}{\varpi} \Psi^{\prime}(u)+\sum_{S \in E_{2}} \frac{-\alpha_{S}}{\varpi} \Psi\left(C^{S}\right)=\sum_{S \in E_{1}} \frac{\alpha_{S}}{\varpi} \Psi\left(C^{S}\right) .
$$

Since $\Psi$ also satisfies (AC), we get

$$
\frac{1}{\varpi} \Psi^{\prime}(u)+\sum_{S \in E_{2}} \frac{-\alpha_{S}}{\varpi} \Psi\left(C^{S}\right)=\frac{1}{\varpi} \Psi(u)+\sum_{S \in E_{2}} \frac{-\alpha_{S}}{\varpi} \Psi\left(C^{S}\right) .
$$

Hence $\Psi^{\prime}(u)=\Psi(u)$, which proves that $\Psi^{\prime}=\Psi$.

Proposition 6.5. The four axioms in Theorem 6.2 are independent.

\section{Proof.}

- The power index $2 \cdot \Psi$ satisfies (NP), (S), (AC), but not (E).

- Denote by ED the equal division power index which assigns $\frac{1}{n}$ to each player for every interval simple game. Then the power index $\frac{1}{2} \cdot \Psi+\frac{1}{2} \cdot$ ED satisfies (E), (S) and (AC), but not (NP). 
- In Proposition 6.1 (c.f. [14, Proposition 4]) we have stated a parametric classes of power indices for interval simple games that satisfy (E), (S), and (NP). In [14] it was also proved that there is at least one parameter a for which the parameterized index $\Psi^{\mathbf{a}} \neq \Psi$. Thus, by Theorem 6.2 we can conclude that $\Psi^{\mathbf{a}}$ does not satisfies (AC). (Also Proposition 5.1 for $(j, k)$ simple games can be adjusted easily.)

- Note that by Proposition 6.4 the set $\left(\widehat{C^{S}}\right)_{S \in 2^{N}}$ is a basis of the vector space of all TU games on $N$. Thus, given an interval simple game $u$, there exists a unique collection of real numbers $\left(y_{S}^{u}\right)_{S \in 2^{N}}$ such that

$$
\widehat{u}=\sum_{S \in 2^{N}} y_{S}^{u} \widehat{C^{S}}
$$

Consider some $i_{0} \in N$ and set

$$
F(u)=\sum_{S \in 2^{N}} y_{S}^{u} \cdot F\left(C^{S}\right)
$$

For each $S \in 2^{N} \backslash\{N\}$ we set $F_{i}\left(C^{S}\right)=\Phi\left(C^{S}\right)$. For $S=N$ we set $F_{i}\left(C^{N}\right)=\frac{2}{n+1}$ if $i=i_{0}$ and $F_{i}\left(C^{N}\right)=\frac{1}{n+1}$ otherwise. We can easily check that $F$ satisfies $(\mathrm{E}),(\mathrm{NP}),(\mathrm{AC})$, but not (S).

This proves that the four axioms in Theorem 6.2 are independent.

\section{Acknowledgment}

Hilaire Touyem benefits from a financial support of the CETIC (Centre d'Excellence Africain en Technologies de l'Information et de la Communication) Project of the University of Yaounde I.

\section{References}

[1] E. Algaba, V. Fragnelli, and J. Sánchez-Soriano. Handbook of the Shapley Value. CRC Press, 2019.

[2] R. Amer, F. Carreras, and A. Magaña. Extension of values to games withmultiple alternatives. Annals of Operations Research, 84:63-78, 1998.

[3] P. Dubey. On the uniqueness of the shapley value. International Journal of Game Theory, 4(3):131$139,1975$.

[4] D. S. Felsenthal and M. Machover. The measurement of voting power. Books, 1998.

[5] J. Freixas. The Shapley-Shubik power index for games with several levels of approval in the input and output. Decision Support Systems, 39(2):185-195, 2005. 
[6] J. Freixas. A value for $j$-cooperative games: some theoretical aspects and applications. In E. Algaba, V. Fragnelli, and J. Sánchez-Soriano, editors, Handbook of the Shapley Value, chapter 14, pages 281-311. CRC Press, 2019.

[7] J. Freixas and W. S. Zwicker. Weighted voting, abstention, and multiple levels of approval. Social Choice and Welfare, 21(3):399-431, 2003.

[8] J. Friedman and C. Parker. The conditional shapley-shubik measure for ternary voting games. Games and Economic Behavior, 108:379-390, 2018.

[9] M. Grabisch, J. Marichal, R. Mesiar, and E. Pap. Aggregation Functions. 2009. Cambridge Univ., Press, Cambridge, UK, 2009.

[10] C.-R. Hsiao and T. Raghavan. Shapley value for multichoice cooperative games, i. Games and Economic Behavior, 5(2):240-256, 1993.

[11] X. Hu. An asymmetric Shapley-Shubik power index. International Journal of Game Theory, $34(2): 229-240,2006$.

[12] S. Kurz. Measuring voting power in convex policy spaces. Economies, 2(1):45-77, 2014.

[13] S. Kurz. Importance in systems with interval decisions. Advances in Complex Systems, 21(6):1850024, 2018.

[14] S. Kurz, I. Moyouwou, and H. Touyem. An axiomatization of the Shapley-Shubik index for interval decisions. arXiv preprint 190\%.01323, 2019.

[15] S. Kurz and S. Napel. The roll call interpretation of the Shapley value. Economics Letters, 173:108$112,2018$.

[16] I. Mann and L. Shapley. The a priori voting strength of the electoral college. In M. Shubik, editor, Game theory and related approaches to social behavior, pages 151-164. Robert E. Krieger Publishing, 1964.

[17] R. Pongou, B. Tchantcho, and N. Tedjeugang. Revenue sharing in hierarchical organizations: A new interpretation of the generalized banzhaf value. Theoretical Economics Letters, 2(4):369-372, 2012. 
[18] L. S. Shapley. A value for $n$-person games. In H. W. Kuhn and A. W. Tucker, editors, Contributions to the Theory of Games, volume 28 of Annals of Mathematical Studies, pages 307-317. Princeton University Press, 1953.

[19] L. S. Shapley and M. Shubik. A method for evaluating the distribution of power in a committee system. American Political Science Review, 48(3):787-792, 1954. 